\title{
Formation, Dissolution, and Transfer Dynamics of a Millimeter-Scale Thin Liquid Droplet in Glycine Solution by Laser Trapping
}

\author{
Ken-ichi Yuyama, ${ }^{\dagger, \ddagger}$ Thitiporn Rungsimanon, ${ }^{\dagger}$ Teruki Sugiyama, ${ }^{*},, \S$ and Hiroshi Masuhara $*, \dagger, \ddagger$ \\ ${ }^{\dagger}$ Graduate School of Materials Science, Nara Institute of Science and Technology, Ikoma 630-0192, Japan \\ ${ }^{\ddagger}$ Department of Applied Chemistry and Institute of Molecular Science, National Chiao Tung University, Hsinchu 30010, Taiwan \\ ${ }^{\S}$ Instrument Technology Research Center, National Applied Research Laboratories, Hsinchu 30076, Taiwan
}

\section{Supporting Information}

ABSTRACT: The formation, dissolution, and transfer of a millimeter-scale dense liquid droplet are demonstrated by focusing a CW near-infrared laser beam into a thin film of glycine solution in heavy water. The entire process is investigated by directly monitoring the temporal change in the two-dimensional surface profile using a laser displacement meter. Upon laser irradiation, the surface depression is initially induced by laser heating, followed by the formation of the shallow convex-shaped droplet around the focal spot, in which the droplet is always in contact with the surrounding
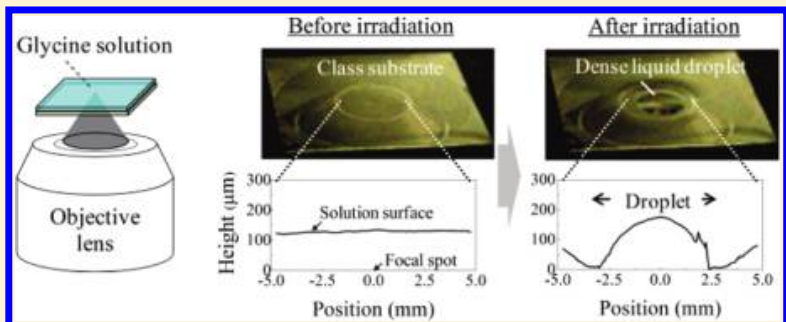

solution through the ultrathin solution layer. After the laser is switched off, the dissolution occurs through the recovery from the ultrathin layer toward the original solution film. When the laser is set to the outside of the droplet, the solution depression is similarly induced, and subsequently the droplet starts moving toward the focal spot. These processes are summarized and discussed in view of laser-induced effects of concentration increase and temperature elevation.

\section{INTRODUCTION}

Laser trapping due to radiation pressure generated by a tightly focused laser beam is a useful method of trapping and manipulating micrometer- and nanometer-sized particles in solution without mechanical contact. This technique is wellknown as optical tweezers and has played innovative roles in many fields of physics, biology, and micromachines. ${ }^{1,2}$ In biological science, for example, this tool is widely used not only for spatial manipulation of various cells but also for observing the kinetic activity of molecular motors at the single-molecule level. $^{3-6}$ For laser trapping of nanoparticles, various research groups, including the current authors, have observed fascinating phenomena of the optical assembly formation of metal and polymer nanoparticles, micelles, and macromolecules, all of which are achieved by accumulating numerous particles and molecules in the focal spot. $^{7-11}$ In an experimental study of macromolecules, the authors successfully trapped repulsive unimolecular micelles with a gyration radius of $5.5 \mathrm{~nm}$ and achieved assembly formation with a $700 \mathrm{~mW}$ continuous wave (CW) laser beam. ${ }^{10}$ Meanwhile, the trapping force for a single small molecule is too weak to overcome its Brownian motion in solution at ambient temperature, so the diffusion of a small dye molecule under radiation pressure is only slightly suppressed in the focal spot. ${ }^{12,13}$ This phenomenon is called "biased diffusion", and a stable trap is not achieved. Note that small molecules forming clusters or aggregates through relatively strong intermolecular interactions can be stably trapped due to their large volume. Once the clusters or aggregates start to assemble in the focal spot, their effective volume increases, inducing stronger radiation pressure. Thus, the local concen- tration increases nonlinearly with time in the focal spot, and the assembly comparable in size to the focal spot is formed. In an earlier study, J-aggregates of pseudoisocyanine dye molecules were successfully deposited in the focal spot by focusing a laser beam into the aqueous solution, and the resultant assembly was fixed on a glass substrate. ${ }^{14} \mathrm{~A}$ recent study for amino acids by Tsuboi et al. also showed that the particle-like assembly can be formed in the focal spot by laser trapping of their clusters. ${ }^{15}$

Laser trapping technique has been also used to induce local phase separation in solution, since it can increase solute concentration at the focal spot by trapping macromolecules and clusters. For example, the current authors demonstrated that a microparticle of poly ( $N$-isopropylacrylamide $)$ with a maximum size of $25 \mu \mathrm{m}$ is formed through phase transition by focusing a laser beam into the aqueous solution. ${ }^{16,17}$ The formation dynamics were discussed in view of laser trapping of polymers and the photothermal effect caused by light absorption of the solution. Later, Delville et al. theoretically investigated these phenomena from the perspectives of radiation pressure and laser heating effects, including convection flow. ${ }^{18}$ Yoshikawa et al. and Kitamura et al. separately reported laser-induced liquid/ liquid phase separation (LLPS) in an oil/water system. ${ }^{19-22}$ Focusing a laser beam into a homogeneous solution produced an oil droplet that grows to several tens of micrometers at the focal spot.

Received: November 3, 2011

Revised: February 24, 2012

Published: February 24, 2012 


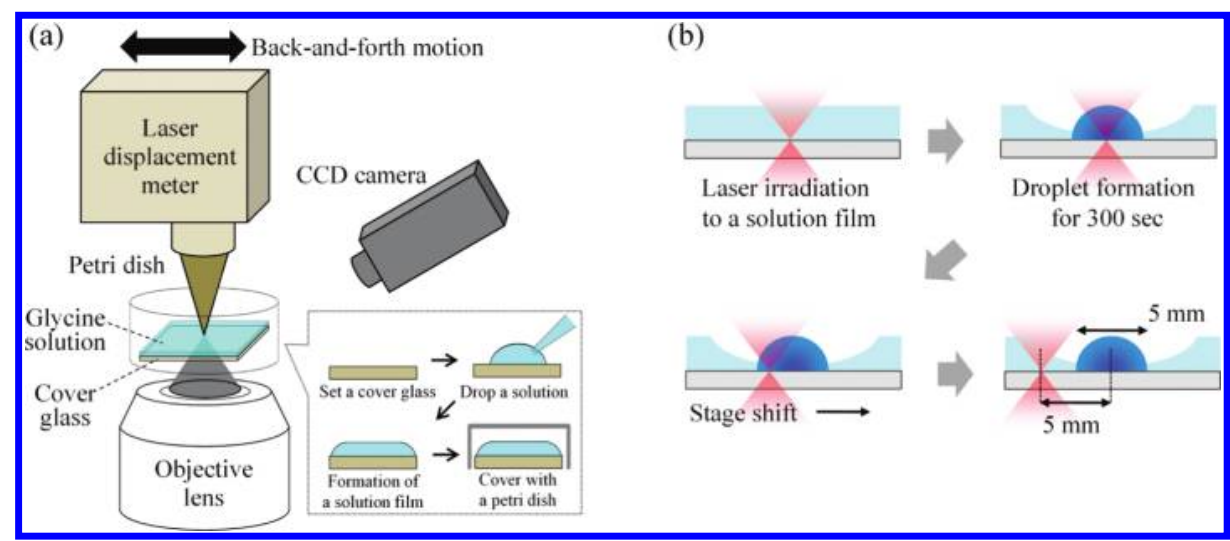

Figure 1. (a) Schematic illustration of the experimental system. (b) Droplet transfer procedure described in section 3.3.

In 2007, we reported the first successful result on laser trapping-induced crystallization of glycine, which can be deemed to be solid/liquid phase separation using a focused laser beam. The crystallization was achieved by laser trapping of the liquid-like clusters at the air/solution interface of the thin film with about $120 \mu \mathrm{m}$ thickness of the supersaturated $\mathrm{D}_{2} \mathrm{O}$ solution. ${ }^{23}$ The authors also confirmed that the crystallization is induced even in the unsaturated solution where no spontaneous crystallization occurs. ${ }^{24}$ It is worth noting that crystallization is achieved only by focusing a laser beam at the air/solution interface, meaning that the crystallization requires not only a high molecular concentration due to laser trapping of the clusters but also a trigger of molecular alignment at the surface layer. We recently demonstrated the formation of a single dense liquid droplet of glycine in its solution by setting the focal spot to the glass/solution interface of the solution thin film. ${ }^{25}$ We explained that the droplet formation occurs through LLPS due to the rapid increase in local concentration caused by laser trapping of the clusters. The characteristic features of this large droplet were summarized as follows: (1) $5 \mathrm{~mm}$ in diameter and $150 \mu \mathrm{m}$ in height, (2) higher molecular concentration compared with the initial supersaturated solution, (3) high stability for about 1 min even after turning off the laser beam, and (4) formation accompanying the dynamic deformation of the solution film. The most remarkable phenomenon is the solution deformation during the droplet formation, which is unlike previous laser-induced phase separation in bulk solution.

In this research, we have investigated the formation and dissolution processes based on temporal change in the twodimensional (2D) surface profile using a confocal displacement meter combined with a microscope. The dynamic profile measurement revealed that an ultrathin solution film was initially formed through the solution depression by laser heating, and then the shallow convex-shaped droplet appeared and grew around the focal spot. The droplet was in contact with the surrounding solution through the ultrathin solution layer. The dissolution process after turning off the laser was also accompanied by the dynamic deformation of the droplet and the surrounding ultrathin layer. We describe these processes in terms of two laser-induced effects, concentration increase and temperature elevation, leading to morphological change in the solution film. We further demonstrate the transfer of the formed droplet and explain it as a combination of formation and dissolution processes.

\section{EXPERIMENTAL SECTION}

Glycine (>99.0\%, Wako) and $\mathrm{D}_{2} \mathrm{O}$ (99.9\%, Cambridge Isotope Laboratories, Inc.) were used as solute and solvent, respectively, and were mixed to obtain a glycine-supersaturated $\mathrm{D}_{2} \mathrm{O}$ solution (23 wt \%, $3.7 \mathrm{~mol} / \mathrm{L}$, supersaturation value; 1.36 ). The preparation details were given in our previous paper. ${ }^{23,25} \mathrm{~A}$ $22 \times 22 \mathrm{~mm}^{2}$ cover glass with a highly hydrophilic surface was placed on the sample stage of an inverted microscope, and then a $40 \mu \mathrm{L}$ portion of the solution was dropped on the substrate. The portion spontaneously spread over the cover glass and formed the solution thin film (thickness; $130 \mu \mathrm{m}$ ). The cover glass was immediately capped by a Petri dish to minimize evaporation of the solvent.

Figure 1a schematically illustrates the setup for this experiment. A CW near-infrared (NIR) laser beam of 1064 $\mathrm{nm}$ from a $\mathrm{Nd}^{3+}: \mathrm{YVO}_{4}$ laser (Spectra Physics, J20-BL-106C) was used as a laser trapping light source and focused at the glass/solution interface of the thin film with the power of $1.1 \mathrm{~W}$ through an objective lens $(60 \times$ magnification, NA $=0.90)$. Droplet formation and transfer during the irradiation were directly monitored from the upper side of the sample using a CCD camera (Flovel, HCC-600). The temporal change in the height of the thin film was measured using a laser confocal displacement meter (Keyence, LT-9030M), ${ }^{25}$ which was also mounted on a computer-controllable motorized stage (Sigma Koki, SGSP20-20). Surface deformation was observed in 2D by moving the displacement meter back and forth to the center of the optical axis at a constant speed of $4.75 \mathrm{~mm} / \mathrm{s}$ in the range of $9.5 \mathrm{~mm}$. The measurement data collected by this device were recorded by a data logger (Keyence, NR-500) every $10 \mathrm{~ms}$.

Figure $1 \mathrm{~b}$ illustrates the droplet transfer procedure described in section 3.3. After a droplet was formed by $300 \mathrm{~s}$ laser irradiation, the focal spot was shifted to the outside of the droplet by moving the microscope stage. The droplet was then observed with a CCD camera while simultaneously measuring the surface height at the focal spot.

\section{RESULTS AND DISCUSSION}

3.1. Laser-Induced Formation Dynamics of a Dense Droplet. Figure 2 shows photographs of the sample set on the microscope stage before and after laser irradiation. Before the irradiation, a cover glass and an objective lens were observed through the solution thin film. After $300 \mathrm{~s}$ laser irradiation, a circular droplet about $5 \mathrm{~mm}$ in diameter was clearly visible on the glass substrate. Droplet formation was reproducibly observed under the present experimental conditions. The 


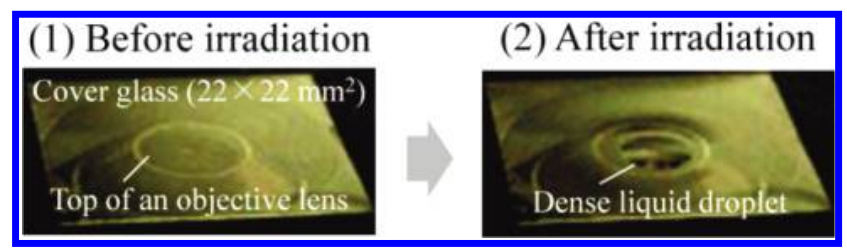

Figure 2. Photographs of a glycine solution film on a $22 \times 22 \mathrm{~mm}^{2}$ cover glass (1) before and (2) after $300 \mathrm{~s}$ laser irradiation. The top of an objective lens is identified through the solution and the cover glass in panel 1 , while a dense liquid droplet is clearly visible in the center of the glass in panel 2 .

formation dynamics of this droplet were investigated by directly measuring the $2 \mathrm{D}$ deformation of the solution during laser irradiation. The deformation in the solution surface was monitored in the central region of the cover glass simultaneously with collecting CCD images from an obliquely upward direction. Figure $3 a$ and $3 b$ shows a series of measured surface profiles and the corresponding CCD camera images, respectively. The origin of the vertical axis in Figure 3a corresponds to the interface between the cover glass and the solution. Before laser irradiation, the solution surface was almost flat in the $9.5 \mathrm{~mm}$ width observation range, and only the top of the objective lens was visible as a dark disk through the solution and the cover glass, as shown in panel 1 of Figure 3a and $3 b$, respectively. The surface depression started immediately after the irradiation. The surface height at and around the focal spot decreased continuously for $15 \mathrm{~s}$, and the solution thickness there gradually became thinner. Consequently, an ultrathin solution film with only $10 \mu \mathrm{m}$ height and in $4 \mathrm{~mm}$ width was formed around the focal spot, as shown in Figure 3a, panel 2, although no apparent change was observed in the CCD image (Figure $3 \mathrm{~b}$, panel 2). The surface depression induced by laser irradiation is well explained as to the decrease in surface tension by local temperature elevation. Further details are given later in this section.

It is interesting to see that further irradiation into the formed ultrathin film made the depressed area swell out, followed by the formation of a shallow convex-shaped droplet, as shown in Figure 3a, panel 3. The 2D profile of Figure 3a, panel 4, showed that the droplet became larger with the laser irradiation. After $60 \mathrm{~s}$ irradiation, the droplet was visible near the focal spot with a CCD camera alone as shown in Figure 3b, panel 4. Eventually at $300 \mathrm{~s}$, it grew larger to a $5 \mathrm{~mm}$ width and a $175 \mu \mathrm{m}$ height clearly visible to the naked eye (panel 5 of Figure $3 a$ and $3 b$ ). Some sharp peaks observed at 1.8 and $2.2 \mathrm{~mm}$ in Figure 3a, panel 5, are ascribed to measurement errors due to the detection limit of the displacement meter. Because the probe light illuminated by the displacement meter is reflected in various directions at steep points of the droplet surface, the intensity of the light returning to the meter becomes weak, resulting in the measurement errors. Nevertheless, one remarkable result is that the formed droplet is in contact with the surrounding solution through an ultrathin solution layer with a few micrometers of height. We successfully made clear the formation dynamics of the glycine dense liquid droplet by utilizing the $2 \mathrm{D}$ surface profile measurement.

The solute concentration of the formed droplet became 1.9 times higher than that of the initial solution (see details in Supporting Information). Because the initial solution has a concentration of $3.7 \mathrm{M}$ (supersaturation value; 1.36), the estimated absolute concentration of the droplet is $7.0 \mathrm{M}$ (supersaturation value; 2.6). Generally, it is quite difficult for a solution of a small organic molecule such as glycine to keep a liquid phase without crystallization at this high supersaturation value. As already suggested in the previous paper, ${ }^{25}$ we presume that the dense droplet is formed through LLPS due to the rapid concentration increase in the liquid-like clusters by their laser trapping. The cluster consists of glycine and $\mathrm{D}_{2} \mathrm{O}$ molecules weakly linked with each other by their intermolecular interactions. The cluster size and shape fluctuate over time, through which the solution keeps a liquid phase without crystallization. Meanwhile, the cooperative rearrangement of glycine molecules inside the cluster leads to nucleation for the crystal. Actually, Myerson et al. investigated the crystallization process in a glycine aqueous solution by means of the smallangle X-ray scattering (SAXS) technique and successfully obtained direct information on the generation processes of the clusters and the subsequent crystal nucleus. ${ }^{26}$ In glycine crystallization by conventional methods, the solute molecules are spontaneously rearranged through the interactions between glycine molecules, leading to the crystal nucleation. In this experiment, the liquid-like clusters should be gathered by the laser irradiation faster than the rate of spontaneous molecular rearrangement inside the clusters, and the internal structure of the clusters possibly remain unchanged during the gathering process. Namely, the extremely high concentration of the clusters is achieved prior to the spontaneous nucleation for the

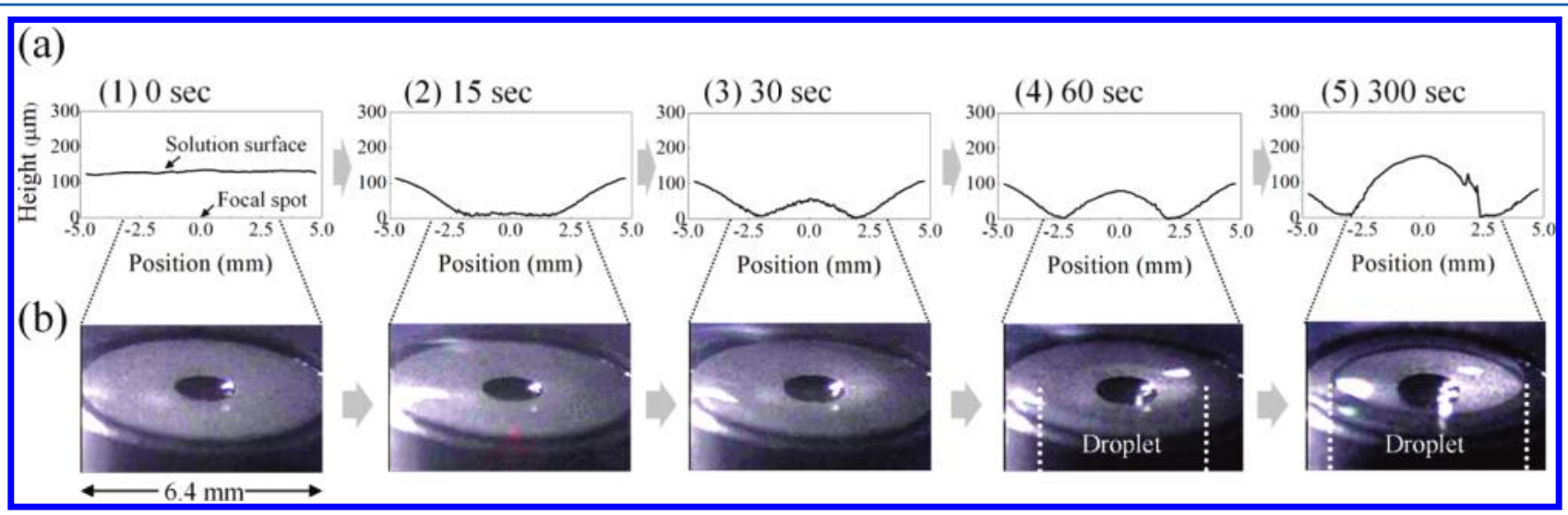

Figure 3. (a) Temporal profile change in a glycine solution film upon focusing the laser beam at a glass/solution interface. Sharp peaks at 1.8 and 2.2 $\mathrm{mm}$ in panel 5 are ascribed to measurement errors. Irradiation time is shown in each profile. (b) The CCD images simultaneously observed with (a). Panels $1-5$ in part $b$ correspond to panels $1-5$ in part a. 


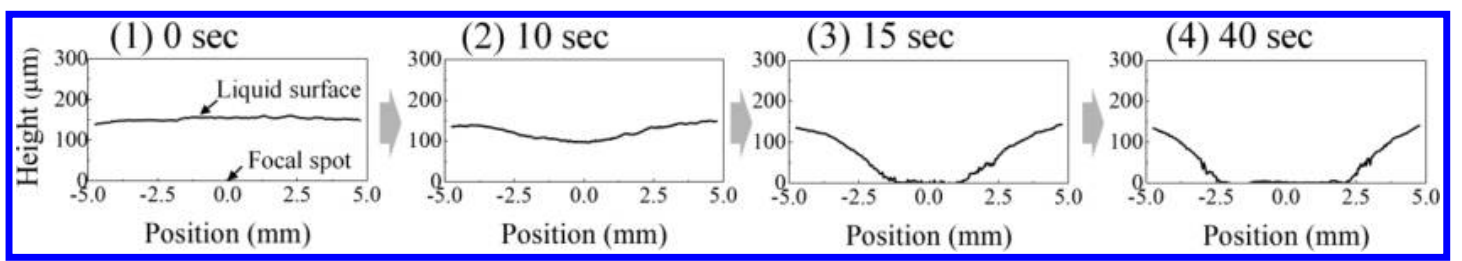

Figure 4. Temporal change in a $\mathrm{D}_{2} \mathrm{O}$ liquid film upon focusing the laser beam at a glass/liquid interface. Irradiation time is shown in each profile.

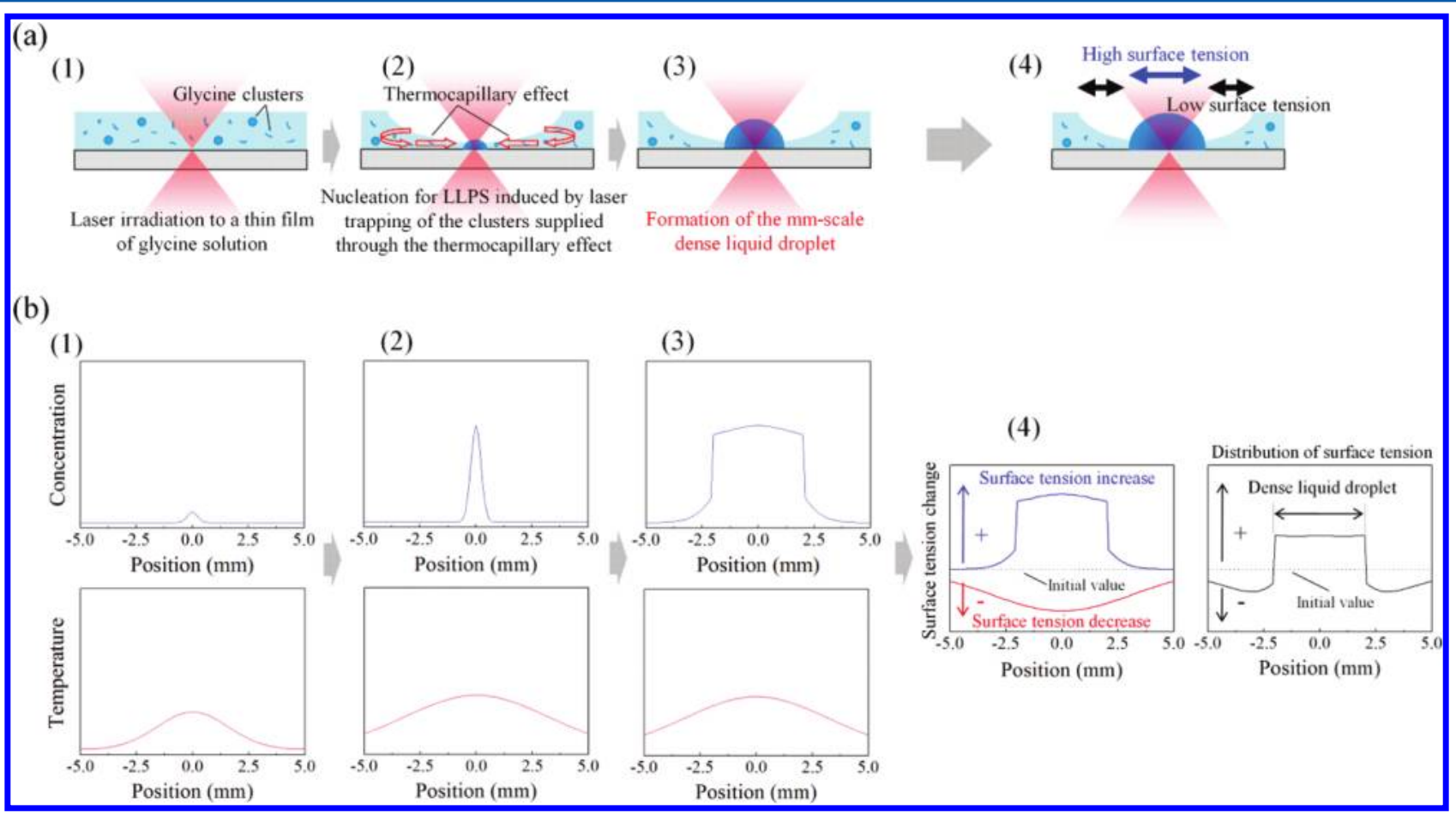

Figure 5. (a) Two-dimensional formation dynamics of a glycine dense liquid droplet. (b) Temporal change in concentration and in temperature distributions under laser irradiation. Total surface energy change during the droplet formation is shown in panel 4.

crystal. Consequently, the association of the clusters containing glycine and $\mathrm{D}_{2} \mathrm{O}$ molecules generates a liquid nucleus, and the millimeter-sized dense liquid droplet is formed by the following growth. $^{27-29}$ On the other hand, crystallization requires two high order parameters of concentration and molecular association structure. ${ }^{28}$ Therefore, the cooperative molecular alignment is only necessary to be given to the formed droplet to trigger the crystallization, because the concentration of the droplet is considerably high. Indeed, shifting the focal spot to the formed droplet surface immediately leads to the crystallization just within a few seconds at the spot. ${ }^{25} \mathrm{We}$ believe that the relatively high order alignment of glycine molecules at the droplet surface assists the crystallization. Further investigation is needed for deeper understanding of the selective nucleation at interfaces.

On the other hand, no droplet was formed in neat $\mathrm{D}_{2} \mathrm{O}$ under the same experimental conditions. Figure 4 shows the temporal change in the surface profile for neat $\mathrm{D}_{2} \mathrm{O}$. At the initial stage of the irradiation, the flat surface started deformation and depression as shown in Figure 4, panels 1 and 2 , which was also observed in the glycine $/ \mathrm{D}_{2} \mathrm{O}$ solution. However, further irradiation continuously depressed the liquid surface until only the glass surface remained. Finally, a rupture was formed around the focal spot in Figure 4, panel 3. The dry area extended and grew rapidly to about $5 \mathrm{~mm}$ wide at $40 \mathrm{~s}$ after irradiation as shown in Figure 4, panel 4. The experiment thus confirmed that the solute glycine is essential for the droplet formation.

The deformation of a liquid film by laser irradiation is generally well-known and has been studied experimentally and theoretically for many years. ${ }^{30-32}$ The liquid surface depression is induced by the local gradient of the surface tension. One of the driving forces in gradient formation is temperature elevation, which decreases the surface tension. The focused laser beam in this experiment causes temperature elevation at the focal spot due to light absorption by both solute and solvent molecules through overtone bands of their molecular vibrations. Subsequently, the temperature gradient is formed through thermal diffusion and convection, leading to the surface depression. Ito et al. estimated the local temperature elevation at the focal spot in neat $\mathrm{D}_{2} \mathrm{O}$ upon trapping by 1064 $\mathrm{nm}$ laser to be $2.6 \mathrm{~K}$ per $1.0 \mathrm{~W} .^{33}$ Furthermore, the $2 \mathrm{D}$ model of surface deformation of a solution thin film, reported by Costa et al., ${ }^{31}$ showed that the temperature elevation needed for depression to the glass surface through the deformation is only $0.4 \mathrm{~K}$ for a neat $\mathrm{D}_{2} \mathrm{O}$ film of $130 \mu \mathrm{m}$ thickness. Under the experimental conditions in the current study, the estimated local temperature elevation of neat $\mathrm{D}_{2} \mathrm{O}$ solution at the focal spot was $3 \mathrm{~K}$. The temperature elevation of the glycine solution film with this concentration was calculated to be $7 \mathrm{~K}$ by a simple comparison of the absorption coefficient of each sample at $1064 \mathrm{~nm}$. The higher temperature elevation in the glycine 


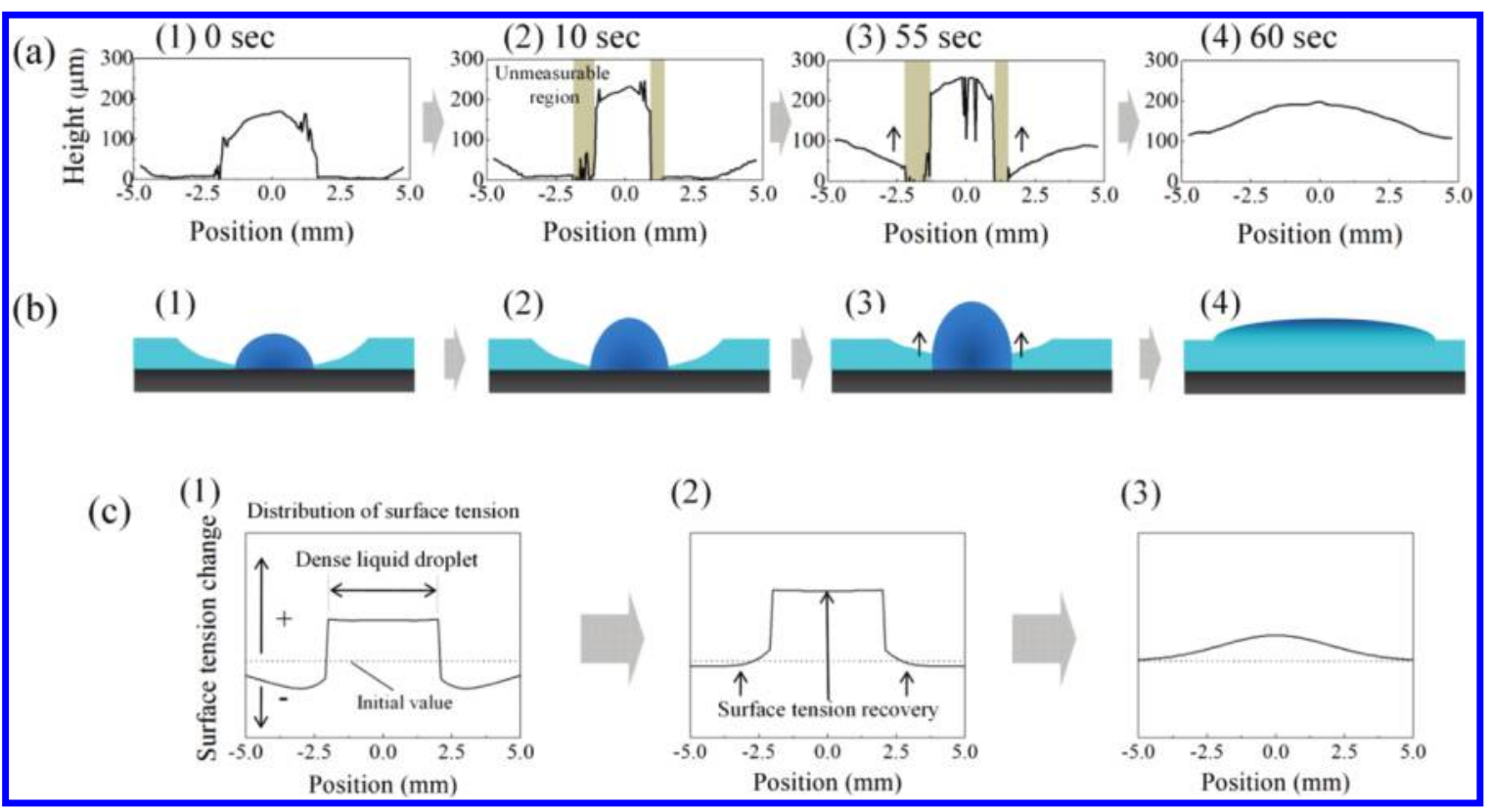

Figure 6. (a) Temporal profile change in a glycine dense liquid droplet and (b) schematic illustrations after turning off the laser beam. The droplet of (a) (panel 1) was prepared by $300 \mathrm{~s}$ laser irradiation. Sharp peaks in (a) are ascribed to measurement errors. Delay time after turning off the laser is shown in each profile. (c) Temporal change in the surface energy in the droplet disappearance process.

solution is ascribed to the much higher absorption coefficient of the glycine molecule compared with that of $\mathrm{D}_{2} \mathrm{O}$. Thus, the temperature gradient induced by laser irradiation at $1.1 \mathrm{~W}$ is enough to cause the surface depression and the following ultrathin film or rupture formation in the glycine $/ \mathrm{D}_{2} \mathrm{O}$ and the neat $\mathrm{D}_{2} \mathrm{O}$ film of $130 \mu \mathrm{m}$ height, respectively.

Here, we discuss the droplet formation dynamics on the basis of surface tension change during laser irradiation. The whole solution deformation in the droplet formation can be explained by two laser-induced effects; the increase in molecular concentration and the temperature elevation. These effects are triggered at the focal spot and extend to the outside area, leading to the surface tension modification of a glycine solution film. Figure 5a schematically illustrates the solution surface which varies from time to time. The corresponding temporal change in the solute concentration and temperature distributions in solution are shown in Figure 5 b.

First, we consider the initial deformation behavior of the glycine $/ \mathrm{D}_{2} \mathrm{O}$ solution in Figure 3a, panels 1 and 2. Upon focusing the laser beam into the solution, its light absorption and laser trapping of the clusters simultaneously occur in the focal spot, inducing the elevation in local temperature accompanied by the surface depression and the increase in local molecular concentration, respectively. Interestingly, the surface depression of the glycine solution was quite similar to that of the neat $\mathrm{D}_{2} \mathrm{O}$ in Figure 4, panels $1-3$, although the temperature elevation in the solution was estimated to be about 2 times higher than that in the pure $\mathrm{D}_{2} \mathrm{O}$. These depression processes should be compared with each other, taking into account some physical properties such as heat conductivity, viscosity, and surface tension. However, the similarity of the depression between the glycine solution and the neat $\mathrm{D}_{2} \mathrm{O}$ strongly supports that, at the initial step of laser irradiation into the solution, temperature elevation is the dominant factor before the local concentration increases (Figure 5b, panel 1). Next, we consider the ultrathin film formation after the initial solution depression as in Figure 5a, panel 2. The main consideration here is the mass transfer effect induced by Marangoni convection flow, namely the thermocapillary effect. Through the effect, the clusters are continuously supplied from the surrounding solution to the depressed area, where radiation pressure works at the central region. The clusters are efficiently trapped in the focal spot one after another, and the trapping force increases due to the large effective volume of their associates. Consequently, a large increase in the concentration of glycine molecules is achieved at and then around the focal spot as shown in the upper graph of Figure $5 b$, panel 2 . Actually, such laser-induced phenomena were reported earlier, ${ }^{34,35}$ in which efficient laser trapping of polymers supplied through the thermocapillary effect was demonstrated in the solution thin film. In parallel with the concentration increase in the focal spot, the local temperature elevation there also becomes accelerated, and the generated heat diffuses to the outer area of the focal spot over time. The lower graph in Figure $5 b$, panel 2, shows the resulting temperature distribution. Generally, the temperature elevation accelerates molecular diffusion, and as a result, molecular assembly by laser trapping should be suppressed. However, the trapping force and the thermocapillary effect cooperatively gather the glycine clusters, and its rate is enough large to compensate the concentration decrease by molecular diffusion due to the temperature elevation. Eventually liquid nucleation to LLPS is possibly achieved. Namely, we can explain that the LLPS is induced by the integration of both laser-induced concentration increase and temperature elevation. After the phase separation, the higher concentration regions grow through concentration fluctuation over time. The concentrated regions have a higher refractive index, and the trapping force should efficiently work on them. Consequently, a gradual concentration distribution is formed as shown in the upper graph of Figure $5 b$, panel 3.

It is reported that the surface tension of glycine aqueous solution increases in increments of about $+0.90 \mathrm{mN} / \mathrm{m}$ per mole with solution concentration. ${ }^{36,37}$ Therefore, the final surface tension of the glycine solution film can be schematically 


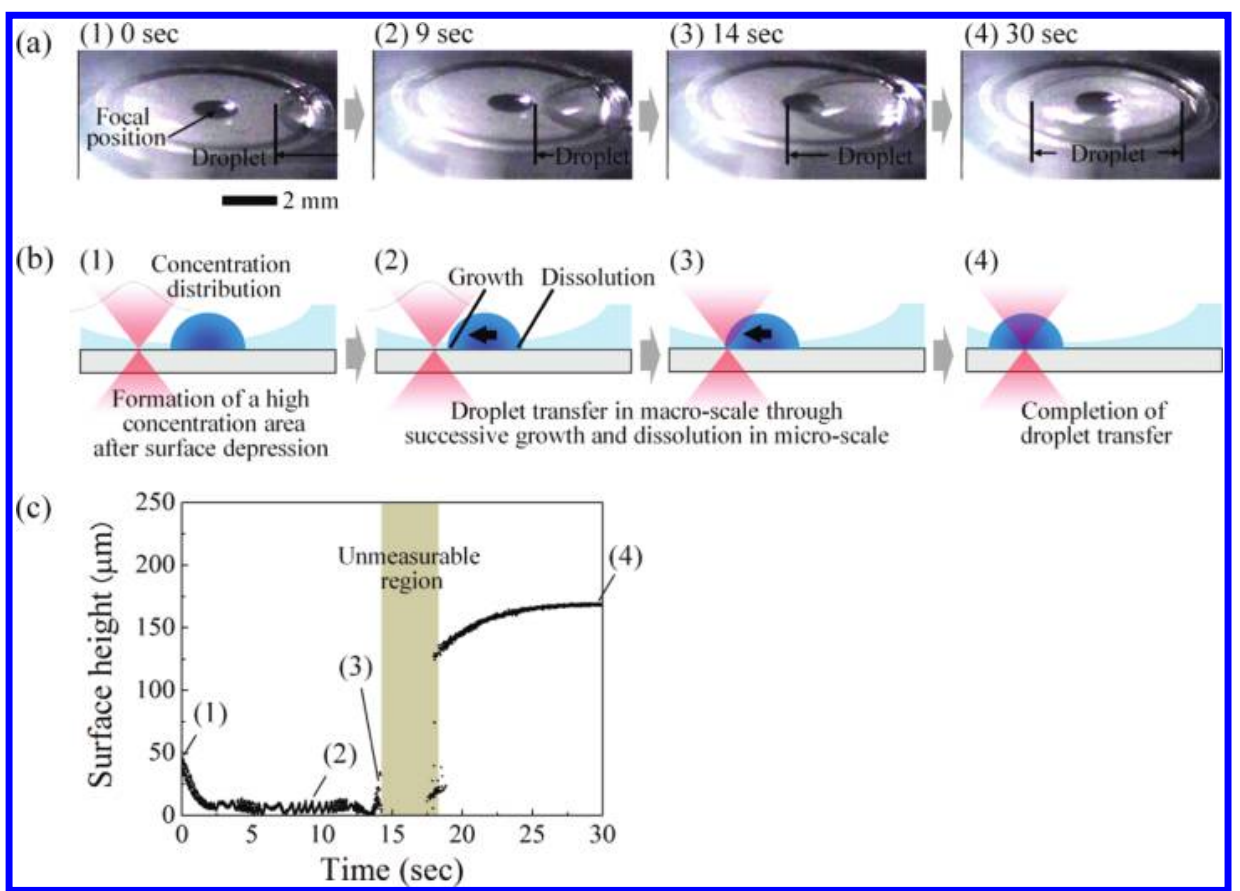

Figure 7. (a) CCD images, (b) schematic illustrations, and (c) surface height change at the focal axis during the droplet transfer. Images and heights of panels 1-4 in parts a and c were observed after applying $0,9,14$, and $30 \mathrm{~s}$ laser irradiation to the outside of the droplet, respectively.

depicted in Figure 5b, panel 4, which is based on the viewpoint of the balance of these concentration and temperature effects. The central area of the thin film has a surface tension much higher than that of the initial solution and the surrounding part. Therefore, the area should tend to conglobate to minimize the surface energy whereas the surrounding solution is predominantly affected by the wide temperature distribution. As a result, the shallow convex-shaped dense liquid droplet and the ultrathin solution film are formed in the center of the thin film and around the droplet, respectively. This result is of great interest because laser irradiation in the micrometer-sized focal spot triggers the bulk phenomenon of the dense droplet formation on a millimeter scale, which is quite similar to nucleation followed by crystallization.

3.2. Dissolution Dynamics of a Dense Droplet. It is important and indispensable for understanding the phase separation to observe and analyze the dissolution process of the formed droplet after turning off the laser beam. The laser was turned off after the droplet was formed by $300 \mathrm{~s}$ laser irradiation. The surface profile measurement for the droplet was then performed as in the above experiment. The start time was set to $0 \mathrm{~s}$ when the laser was turned off. Immediately after switching off the laser at $0 \mathrm{~s}$, no apparent shape change was observed (panel 1 of Figure $6 \mathrm{a}$ and $6 \mathrm{~b}$ ). After $10 \mathrm{~s}$, the droplet vertical length increased as shown in panel 2 of Figure $6 a$ and $6 \mathrm{~b}$. However, the edge of the droplet could not be measured precisely due to the steep slope at the droplet surface. After the laser is terminated, the temperature distribution gradually homogenizes due to thermal diffusion, while the concentration in the droplet may be kept for a while. Figure $6 \mathrm{c}$ summarizes the dissolution behavior in view of the surface tension change. The decrease in the surface tension due to temperature elevation gradually recovers after the laser is terminated, so the tension of the droplet increases as shown in Figure 6c, panels 1 and 2 . Thus, the droplet tends to be more spherical to minimize the surface energy, increasing the height in the center of the droplet. Simultaneously, the ultrathin solution layer around the droplet started recovering from outside due to spatial homogenization of the surface tension (panel 3 of Figure 6a and $6 \mathrm{~b}$ ). When the droplet reaches the surrounding solution through the recovery from the ultrathin layer toward the original solution film, the mass transfer toward the droplet is attenuated. Namely, the opposite molecular diffusion toward the surrounding solution becomes dominant, and the droplet dissipates.

Interestingly, even after the disappearance of the droplet, the solution surface exhibited a slight bulge as shown in panel 4 of Figure $6 \mathrm{a}$ and $6 \mathrm{~b}$. This result strongly suggests that the highly concentrated area with high surface tension should remain for a while as illustrated in Figure 6c, panel 3. The absolute recovery to the initial flat surface took about $3 \mathrm{~min}$ after the droplet disappearance in the CCD observation. It was reported that the diffusivity of glycine in the aqueous solution gradually becomes lower as the concentration increases, and dramatically decreases in the supersaturated region. ${ }^{38}$ On the basis of the report, the diffusion coefficient in the droplet (supersaturation value; 2.6) can be calculated to be almost zero by assuming the linear relation between the diffusivity and the concentration in the supersaturated condition. Practically, the result of Figure 6a, panel 4, would mean that the molecular diffusion is much suppressed by the strong solute-solute or solute-solvent interactions in the droplet. That is, the cluster structure inside the droplet might remain for a certain time through the interactions even after the droplet disappears. The feature prompts us to investigate the molecular association structures in the droplet from viewpoints of spectroscopic and X-ray crystallographic analyses in the future.

3.3. Laser-Controlled Transfer of a Dense Droplet. The high stability of the droplet without laser irradiation enabled us to transfer the droplet by controlling the focal position. Figure $7 \mathrm{a}$ and $7 \mathrm{~b}$ shows a series of CCD images of the transfer behavior (see movie 1 in Supporting Information) and the 
schematic illustrations, respectively. The laser focus shown in Figure $7 \mathrm{a}$, panel 1 , was positioned at $5 \mathrm{~mm}$ away from the center of the droplet visible on the right side. Several seconds later, the droplet started moving toward the focal position and proceeded on the trajectory as shown in Figure 7a, panels 2 and 3. After the droplet edge reached the optical axis, the transfer rate gradually slowed, and the transfer process was completed when the center of the droplet overlapped with the axis of the laser beam (Figure 7a, panel 4). We consider that the transfer consists of a few different processes and try to explain the dynamics in view of a combination of formation and dissolution processes.

Figure $7 \mathrm{c}$ shows the change in surface height at the focal point during the droplet transfer. The initial height was $45 \mu \mathrm{m}$ as shown in Figure 7c, panel 1, which was formed in the droplet formation process. Note that the surface height decreased from $45 \mu \mathrm{m}$ to several micrometers immediately after irradiation, and then the droplet started moving. This solution deformation behavior is quite similar to the initial stage of the droplet formation described in section 3.1, although no new droplet is formed. In relation to the droplet transfer, we refer to the phenomena of crystal growth and dissolution induced by laser trapping. ${ }^{39}$ In those experiments, upon focusing a laser beam closely to a glycine crystal generated spontaneously, the crystal growth was immediately induced, but no new crystal was formed. Similarly three crystals were positioned around the focal spot, where one crystal grew to a larger size, and the others became smaller by dissolution. That is, Ostwald ripening is induced under the laser irradiation. The crystal growth is achieved by consuming the clusters gathered by laser trapping, while glycine molecules used for the growth are supplied by dissolution of other crystals. Coming back to the droplet transfer, those results on the crystal growth and dissolution enable us to explain the transfer mechanism from the viewpoints of the droplet growth and dissolution, as shown in Figure $7 \mathrm{~b}$. First, the high concentration area is formed by the integration of laser trapping of the clusters and the thermocapillary effect as similar to the droplet formation (Figure $7 \mathrm{~b}$, panel 1). As the area continuously extends and eventually reaches the closest edge part of the droplet, the droplet grows toward the focal spot by using the solutes in the area. Simultaneously, the amount of the solutes used for the growth should correspond to that dissolved from the other edge parts of the droplet as shown in Figure $7 b$, panel 2. As a result, the droplet transfer is achieved by the repetition of its growth and dissolution at the near and far edge parts of the droplet from the focal spot, respectively, as depicted in Figure $7 \mathrm{~b}$, panel 3. After the optical axis overlaps with the edge of the droplet, the geometric trapping force for a bulk droplet possibly works dominantly, ${ }^{40}$ and the transfer rate becomes slow. Looking at the variation of rate depending on the droplet position, some kinds of convection flow toward the focal spot may be another driving force for the droplet transfer. ${ }^{41-43}$ Thus, the successive millimeter-scale transfer is achieved through microscale droplet growth and dissolution. Interestingly, this transfer process also requires the formation of the ultrathin film, which produces extremely high concentration area by the integration of laser trapping due to radiation pressure and the thermocapillary effect.

\section{CONCLUSION}

The formation, dissolution, and transfer of a millimeter-scale dense liquid droplet of glycine were achieved by irradiating a focused laser beam into the solution thin film. These processes were investigated by directly observing the temporal change in the $2 \mathrm{D}$ solution surface profile with a laser displacement meter and a CCD camera. In the initial stage of droplet formation, the ultrathin solution film with $10 \mu \mathrm{m}$ was formed through the surface depression due to laser heating. An effective mass transfer due to the thermocapillary effect was induced in the depressed area, where the trapping force worked at the central region. Spatial integration of the mass transfer and the trapping force then resulted in high concentration, leading to the liquid nucleation to LLPS. The shape of the droplet and its surrounding solution gave us remarkable viewpoints. The formed droplet was in contact with the surrounding solution through the ultrathin solution layer. The dissolution of the droplet occurred after terminating the laser beam, which could be interpreted in terms of the mass transfer direction in the ultrathin solution layer. The transfer of the droplet itself was started in the ultrathin film through which the mass transfer takes place. Thus, the formation of the ultrathin solution layer played a significant role in the all processes of formation, dissolution, and transfer of the droplet. This ultrathin film formation was eventually ascribed to two laser-induced effects, namely, concentration increase and temperature elevation, working cooperatively around the focal spot. We now understand that intense laser irradiation into a molecular solution under a microscope leads to spatial and temporal changes in not only its physical and chemical properties but also its mechanical shapes. Furthermore, it is worth noting that the droplet formation and transfer on a millimeter scale is controlled by the laser irradiation on a micrometer scale. This spatial amplification will provide us a new viewpoint for controlling spatial-temporal distribution of molecular concentration in solution, and the relevant experimental techniques should contribute significantly to the research on liquid/liquid and solid/liquid phase separation. We believe that the resultant findings will be a milestone toward a new area of laser trapping chemistry.

\section{ASSOCIATED CONTENT}

\section{S Supporting Information}

Details of the estimation of solute concentration in the droplet and a movie of the droplet transfer. This material is available free of charge via the Internet at http://pubs.acs.org.

\section{AUTHOR INFORMATION}

\section{Corresponding Author}

*E-mail: sugiyama@itrc.narl.org.tw (T.S.); masuhara@ masuhara.jp (H.M.).

\section{Notes}

The authors declare no competing financial interest.

\section{ACKNOWLEDGMENTS}

The present work is partly supported by a KAKENHI (S) grant (a Grant-in-Aid for Scientific Research) (No. 18106002) from the Japan Society for the Promotion of Science (JSPS) to H.M., the MOE-ATU Project (National Chiao Tung University) of the Ministry of Education, Taiwan, to H.M., the National Science Council of Taiwan (No. 100-2113-M-009-001) to H.M., (NSC 100-2113-M-492-002-MY2) to T.S., a KAKENHI grant on Priority Areas "Strong Photon-Molecule Coupling Fields" from the Ministry of Education, Culture, Sports, Science and Technology of Japan (MEXT) (No. 21020022) to T.S., a 
KAKENHI (C) grant (No. 20550136) to T.S. Dr. Anwar Usman, who is our colleague, is appreciated for his kind advice in English writing.

\section{REFERENCES}

(1) Ashkin, A. Proc. Natl. Acad. Sci. U.S.A. 1997, 94, 4853-4860.

(2) Grier, D. G. Nature 2003, 424, 810-816.

(3) Ashkin, A.; Dziedzic, J. M. Science 1987, 235, 1517-1520.

(4) Ashkin, A.; Dziedzic, J. M.; Yamane, T. Nature 1987, 330, 769771.

(5) Kuo, S. C.; Sheetz, M. P. Science 1993, 260, 232-234.

(6) Svoboda, K.; Schmidt, C. F.; Schnapp, B. J.; Block, S. M. Nature 1993, 365, 721-726.

(7) Hosokawa, C.; Yoshikawa, H.; Masuhara, H. Phys. Rev. E 2004, 70, 0614101-7.

(8) Tanaka, Y.; Yoshikawa, H.; Itoh, T.; Ishikawa, M. Opt. Express 2009, 17, 18760-18767.

(9) Hotta, J.; Sasaki, K.; Masuhara, H. J. Am. Chem. Soc. 1996, 118, 11968-11969.

(10) Hotta, J.; Sasaki, K.; Masuhara, H.; Morishima, Y. J. Phys. Chem. B 1998, 102, 7687-7690.

(11) Singer, W.; Nieminen, T. A.; Heckenberg, N. R.; RubinszteinDunlop, H. Phys. Rev. E 2007, 75, 011916 1-5.

(12) Osborne, M. A.; Balasubramanian, S.; Furey, W. S.; Klenerman, D. J. Phys. Chem. B 1998, 102, 3160-3167.

(13) Chirico, G.; Fumagalli, C.; Baldini, G. J. Phys. Chem. B 2002, 106, 2508-2519.

(14) Tanaka, Y.; Yoshikawa, H.; Asahi, T.; Masuhara, H. Appl. Phys. Lett. 2007, 91, 041102 1-3.

(15) Tsuboi, Y.; Shoji., T.; Kitamura, N. J. Phys. Chem. C 2010, 114, 5589-5593.

(16) Hofkens, J.; Hotta, J.; Sasaki, K.; Masuhara, H.; Iwai., K. Langmuir 1997, 13, 414-419.

(17) Ishikawa, M.; Misawa, H.; Kitamura, N.; Fujisawa, R.; Masuhara, H. Bull. Chem. Soc. Jpn. 1996, 69, 59-66.

(18) Delville, J. P.; Lalaude, C.; Ducasse, A. Physica A (Amsterdam, Neth.) 1999, 262, 40-68.

(19) Mukai, S.; Magome, N.; Kitahara, H.; Yoshikawa, K. Appl. Phys. Lett. 2003, 83, 2557-2559.

(20) Mukai, S.; Kitahara, H.; Yoshikawa, K. Chem. Phys. Lett. 2005, 402, 529-534.

(21) Sadakane, K.; Kitahara, H.; Seto, H.; Yoshikawa, K. Phys. Rev. E 2008, 78, 0462141-4.

(22) Kitamura, N.; Yamada, M.; Ishizaka, S.; Konno, K. Anal. Chem. 2005, 77, 6055-6061.

(23) Sugiyama, T.; Adachi, T.; Masuhara, H. Chem. Lett. 2007, 36, $1480-1481$.

(24) Rungsimanon, T.; Yuyama, K.; Sugiyama, T.; Masuhara, H. Cryst. Growth Des. 2010, 10, 4686-4688.

(25) Yuyama, K.; Sugiyama, T.; Masuhara, H. J. Phys. Chem. Lett. 2010, 1, 1321-1325.

(26) Chattopadhyay, S.; Erdemir, D.; Evans, J. M. B.; Ilavsky, J.; Amenitsch, H.; Segre, C. U.; Myerson, A. S. Cryst. Growth Des. 2005, 5, 523-527.

(27) He, G.; Tan, R. B. H.; Kenis, P. J. A.; Zukoski, C. F. J. Phys. Chem. B 2007, 111, 14121-14129.

(28) Vekilov, P. G. Cryst. Growth Des. 2004, 4, 671-685.

(29) Bonnett, P. E.; Carpenter, K. J.; Dawson, S.; Davey, R. J. Chem. Commun. 2003, 698-699.

(30) Costa, G. D.; Calatroni, J. Appl. Opt. 1978, 17, 2381-2385.

(31) Costa, G. D.; Calatroni, J. Appl. Opt. 1979, 18, 233-235.

(32) Gugliotti, M.; Baptista, M. S.; Politi, M. J. Langmuir 2002, 18, 9792-9798.

(33) Ito, S.; Sugiyama, T.; Toitani, N.; Katayama, G.; Miyasaka, H. J. Phys. Chem. B 2007, 111, 2365-2371.

(34) Louchev, O. A.; Juodkazis, S.; Murazawa, N.; Wada, S.; Misawa, H. Opt. Express 2008, 16, 5673-5680.
(35) Nabetani, Y.; Yoshikawa, H.; Grimsdale, A. C.; Müllen, K.; Masuhara, H. Langmuir 2007, 23, 6725-6729.

(36) Pappenheimer., J. R.; Lepie, M. P.; Wyman, J. Jr. J. Am. Chem. Soc. 1936, 58, 1851-1855.

(37) Weissbuch., I.; Frolow, F.; Addadi, L.; Lahav, M.; Leiserowitz, L. J. Am. Chem. Soc. 1990, 112, 7718-7724.

(38) Chang, Y. C.; Myerson, A. S. AIChE J. 1986, 32, 1567-1569.

(39) Sugiyama, T.; Adachi, T.; Masuhara, H. Chem. Lett. 2009, 38, $482-483$

(40) Masuo, S.; Yoshikawa, H.; Asahi, T.; Masuhara, H.; Sato, T.; Jiang, D.; Aida, T. J. Phys. Chem. B 2002, 106, 905-909.

(41) Delville, J.; Vincent, M. R. S.; Schroll, R. D.; Chraibi, H.; Issenmann, B.; Wunenburger, R.; Lasseux, D.; Zhang, W. W.; Brasselet, E. J. Opt. A: Pure Appl. Opt 2009, 11, 034015 1-15.

(42) Diguet, A.; Guillermic, R.; Magome, N.; Saint-Jalmes, A.; Chen, Y.; Yoshikawa, K.; Baigl, D. Angew. Chem., Int. Ed. 2009, 48, 92819284.

(43) Rybalko, S.; Magome, N.; Yoshikawa, K. Phys. Rev. E 2004, 70, $0463011-4$. 